\title{
Relationship between ambulatory physical activity assessed by activity trackers and physical frailty among nursing home residents.
}

\author{
Bruyère $\mathrm{O}^{1,2 \& 3}$, Reginster $\mathrm{JY}^{1,2}$, Dardenne $\mathrm{N}^{1,2}$, Nelis $\mathrm{J}^{3}$, Lambert $\mathrm{E}^{3}$, Appelboom $\mathrm{G}^{4}$, Buckinx \\ $\mathrm{F}^{1,2}$
}

1 Department of Public health, Epidemiology and health Economics, University of Liège, Liège, Belgium.

2 Support Unit in Epidemiology and Biostatistics, University of Liège, Liège, Belgium.

3 Department of Motricity Sciences, University of Liège, Liège, Belgium.

4 Neuro-digital Initiative, Columbia University, New York, USA.

Backgrounds: The aim of this study was to assess the relationship between the level of ambulatory physical activity, measured using a physical activity tracker, and the clinical components of physical frailty, among nursing home residents.

Methods: we proceeded in 3 steps:

The first step was the validation of the physical activity tracker (i.e. the Pebble): volunteer adults walked 3 times 15 minutes on a treadmill at three different speeds $(2.5 \mathrm{~km} / \mathrm{h}, 4 \mathrm{~km} / \mathrm{h}$ and $5.5 \mathrm{~km} / \mathrm{h}$ ). The number of steps was recorded by 2 Pebble devices placed on the subjects (one at the foot level and the other one at the hip level). The number of steps registered by the Pebble was then compared, with the real number of steps counted by two investigators. Intraclass correlation coefficients (ICC) were then calculated.

The second step was the measurement of ambulatory physical activity, using the Pebble trackers, over a 7-day period, among nursing home residents: volunteer nursing home residents wore the activity tracker on their shoes for seven consecutive days.

The third step consisted in studying the relationship between the results obtained with the Pebble trackers (in step 2) and clinical characteristics, linked to physical frailty, of the subjects: a large number of clinical data had been collected for the subjects who wore a Pebble during step 2: body mass index (BMI), energy expenditure (Minnesota questionnaire), cognitive status (MMSE), nutritional status (MNA), quality of life (SF-36), level of autonomy (Katz), functional and motors skills (Timed Up and Go test, Tinetti test, Short Physical Performance Battery (SPPB)), fear of falling (FES-1), peak flow, grip strength, isometric strength of 8 different muscle groups (MicroFET2), frailty status (Fried), and body composition (Bioelectrical Impedance Analyzer, the InBody S10). These data were compared according to the level of ambulatory physical activity of the patient.

Results: For the first step, the validation of the Pebble devices, 24 subjects were included (12 young students aged between 21 and 30 years and 12 adults aged over 65 years). ICC data showed that the reliability of the Pebble, whatever the tested speed, is better when placed on the foot level. Under this condition, the ICC of the Pebble tracker assessing its reliability varied from 0.60 to 0.93 depending of the tested speed. 
For the second step, 27 nursing home subjects aged $86.7 \pm 7.81$ years were included in the study in order to measure the ambulatory physical activity, over a 7-day period. On average, residents walked $1678.4 \pm 1621$ (median $=1300)$ steps per day.

The last step aimed to assess the relationship between the results obtained with the Pebble trackers and clinical characteristics, linked to physical frailty, of the subjects. As it could be expected, the energy expenditure, estimated by the Minnesota questionnaire, was significantly higher ( $\mathrm{p}=0.0003$ ) among people who walked more than 1300 steps per day than in those who walked less than 1300 steps a day. These subjects had also a significantly higher MMSE score $(\mathrm{p}=0.005)$, Katz level $(\mathrm{p}=0.04)$, Tinetti score (0.0003), SPBB score $(\mathrm{p}=0.002)$, peak flow ( $\mathrm{p}=0.001$ ), isometric strength of the 8 muscle groups tested ( $\mathrm{p}$-value ranged from 0.0003 to $0.01)$ and grip strength $(\mathrm{p}=0.003)$ as well as a significantly lower time required to perform the Timed up and Go test (0.0004) than subjects who walked less than 1300 steps per day. Moreover, subjects more active (i.e. $>1300$ steps/day) were significantly less frail (i.e. Fried score) than less active subjects $(\mathrm{p}=0.0005)$. No other significant difference was observed between the two groups.

Conclusion: This study showed that ambulatory physical activity of nursing home residents, assessed using a physical activity tracker, is lower than currently recommended in the elderly. Lower ambulatory physical activity is associated with poorer muscular and physical performances. 